\title{
The Impartial, Anonymous and Neutral Culture Model: A Probability Model for Sampling Public Preference Structures
}

\author{
Ömer Ĕ̆gecioğlu \\ Department of Computer Science, \\ University of California, Santa Barbara, \\ CA 93106, USA \\ omer@cs.ucsb.edu \\ Ayça E. Giritligil \\ Institute of Social Sciences \\ Istanbul Bilgi University \\ Dolapdere Campus \\ Istanbul 34440, TURKEY \\ aebru@bilgi.edu.tr
}

\begin{abstract}
We introduce a new probability model, namely Impartial, Anonymous and Neutral Culture model (IANC), for sampling public preferences concerning a given set of alternatives. IANC treats public preferences through a class of preference profiles named roots, where both names of the voters and of the alternatives are immaterial. The general framework along with the theoretical formulation through group actions, an exact formula for the number of roots, and the description of a symbolic algebra package that allows for the generation of roots uniformly are presented. In order to be able to obtain uniform distribution of roots for large electorate size and high number of alternatives which lead to combinatorial explosions, the machinery we developed involves elements of symmetric functions and an application of the Dixon-Wilf algorithm. Using Monte-Carlo methods, the model we develop allows for a testbed that can be used to answer various questions about the properties and behaviors of anonymous and neutral social choice rules for large parameters. As applications of the method, the results of two MonteCarlo experiments are presented: the likelihood of the existence of Condorcet winners, and the probability of Condorcet and Plurality rules to choose the same winner.
\end{abstract}




\title{
The Impartial, Anonymous and Neutral Culture Model: A Probability Model for Sampling Public Preference Structures
}

\begin{abstract}
We introduce a new probability model, namely Impartial, Anonymous and Neutral Culture model (IANC), for sampling public preferences concerning a given set of alternatives. IANC treats public preferences through a class of preference profiles named roots, where both names of the voters and of the alternatives are immaterial. The general framework along with the theoretical formulation through group actions, an exact formula for the number of roots, and the description of a symbolic algebra package that allows for the generation of roots uniformly are presented. In order to be able to obtain uniform distribution of roots for large electorate size and high number of alternatives which lead to combinatorial explosions, the machinery we developed involves elements of symmetric functions and an application of the Dixon-Wilf algorithm. Using Monte-Carlo methods, the model we develop allows for a testbed that can be used to answer various questions about the properties and behaviors of anonymous and neutral social choice rules for large parameters. As applications of the method, the results of two MonteCarlo experiments are presented: the likelihood of the existence of Condorcet winners, and the probability of Condorcet and Plurality rules to choose the same winner.
\end{abstract}

\section{Introduction}

In social choice theory, all procedures aggregating individual preferences into social choice are analyzed in the light of some "robustness" and "reliability" criteria, such as non-vulnerability to various paradoxes, monotonicity, consistency, strategy-proofness, self-selectivity, etc. Theoretically, a single concrete example is enough to conclude that a social choice rule (SCR) fails in any of these criteria. However, it is also of interest to know the likelihood of such a problem occurring in practice and to detect the "behavior" patterns of a SCR with respect to alteration of the number of voters and alternatives.

An extensive literature has been devoted to analyzing the properties and behaviors of various SCRs through probability models designed to generate voters' preferences. There are two basic models in this literature, namely, Impartial Culture (IC) and Impartial Anonymous Culture(IAC) models. IC uses preference profiles, which show how each of $n$ voters in an electorate totally orders (linearly ranks) $m$ alternatives, for generating voters' preferences, and regards each preference profile equally likely. IAC, on the other hand, is based on the presentation of voter preferences by anonymous profiles where the names of the voters are neglected, and assumes that each resulting anonymous profile class is equally probable. 
In this paper, we introduce the Impartial, Anonymous and Neutral Culture (IANC) model, which treats voter preferences through a class of preference profiles where not only the names of the voters, but also the names of the alternatives are immaterial. This approach reflects two basic axioms of social choice theory: Anonymity and Neutrality. Anonymity requires voters to be treated equally whereas neutrality prohibits a SCR from having a built-in bias for or against any one or more alternatives. In other words, if the names of the voters and/or alternatives are permuted, the winner(s) of the aggregation process induced by any anonymous and neutral SCR remain(s) intact except from the corresponding alternative names after permutation. Hence, given the number of alternatives and voters, some preference profiles are "equal" to each other from the perspective of anonymous and neutral SCRs, since they can be generated from each other via relabeling alternatives and voters. Therefore, such SCRs lead to a partition of preference profiles into anonymous and neutral equivalence classes. Any preference profile in such an anonymous and neutral equivalence class can be taken as the representative profile which we refer to as a root. Thus, each root represents a structurally distinct preference profile under simultaneous fulfillment of anonymity and neutrality axioms.

To our knowledge, this is the first study in the literature which introduces a formulation of roots for given number of alternatives and voters. The notion of a root itself (as a core preference structure independent of the names of the alternatives and of the voters) appeared first in Sertel and Giritligil $[10,16]$. In these experimental studies, the subjects are confronted with hypothetical preference of an hypothetical electorate over some abstract set of alternatives at which several SCRs of focus choose distinct winners. The subjects are shown all possible roots that can be generated fulfilling this constraint, and being confronted with each root, the subjects are asked which of the alternatives should be chosen for that society. As the "veil of ignorance" is provided in the experimental setting, the answers of the subjects show their "democratic" values about how to aggregate individual preference into a social choice. Note that in experimental studies of this kind, it is very important to be aware of the set of all possible roots to sample from. Random sampling of preference profiles instead of roots is surely prone to biased and incomplete data since the relative position of the winner of a SCR can be "favorable" in some roots and can be "unfavorable" in some others. That is, a random sampling from preference profiles can create bias for or against some SCRs. Hence, it is crucial to consider (and carefully sample from in a computational approach) all possible preference structures when the voting outcomes are analyzed.

As we introduce a group theoretic formulation of roots, we describe a symbolic algebra package that allows for the generation of roots from the uniform distribution. By the Monte-Carlo method, this black-box model allows for a testbed that can be used to answer various questions about the properties of anonymous and neutral SCRs experimentally. Since there is a combinatorial explosion for large values of $m$ and $n$, any simple enumeration of roots is definitely insufficient to select representatives uniformly. We use ideas from the theory of symmetric functions and specialize the general Dixon-Wilf algorithm to overcome this problem. 


\section{Basic notions and definitions}

\subsection{Preference profiles and equivalence classes}

We consider a finite collection of voters $\{1,2, \ldots, n\}$. Each voter is assumed to have a total (linear) preference order (a complete, anti-symmetric and transitive binary relation) on a finite set of alternatives $A=\left\{a_{1}, a_{2}, \ldots, a_{m}\right\}$. A preference profile $P$ is an $n$-tuple of total orders on $A$. Clearly, for $m$ alternatives and $n$ voters, there are $m !^{n}$ preference profiles.

Let us represent a preference profile as an $m \times n$ matrix which shows how each of the $n$ voters linearly ranks $m$ alternatives. We assume that the voters correspond to the columns and the alternatives correspond to the rows of the matrix. In this representation, the entries in the $j$-th column of the matrix lists the preferences of the $j$-th voter in decreasing rank from the first row down to the last row. In particular the first row of the matrix consists of the entries from $A$ that are the voters' top-ranked alternatives.

As an example, consider a case with two alternatives, $a_{1}$ and $a_{2}$, and three voters whose preferences over $a_{1}$ and $a_{2}$ are given in sequential columns. There are two possible linear preference rankings for two alternatives: $a_{1}$ is strictly preferred to $a_{2}$, or $a_{2}$ is strictly preferred to $a_{1}$. In this case there is a total of $(2 !)^{3}=8$ preference profiles.

$$
\begin{aligned}
& P_{1}=\begin{array}{|l|l|l|}
\hline a_{1} & a_{1} & a_{1} \\
\hline a_{2} & a_{2} & a_{2} \\
P_{5}=
\end{array} \quad P_{2}=\begin{array}{|l|l|l|l|}
a_{1} & a_{1} & a_{2} \\
\hline a_{2} & a_{2} & a_{1} \\
\hline a_{2} & a_{2} & a_{1} \\
\hline a_{1} & a_{1} & a_{2} \\
\hline
\end{array} \quad P_{6}=\begin{array}{|l|l|l|l|l|l|}
\hline a_{2} & a_{1} & a_{2} \\
\hline a_{1} & a_{2} & a_{1} \\
\hline a_{2} & a_{1} & a_{2} \\
\hline
\end{array} \quad P_{7}=\begin{array}{|l|l|l|l|}
\hline a_{1} & a_{2} & a_{2} \\
\hline a_{2} & a_{1} & a_{1} \\
\hline a_{1} & a_{2} & a_{2} \\
\hline a_{2} & a_{1} & a_{1} \\
\hline a_{2} & a_{2} & a_{2} \\
\hline a_{1} & a_{1} & a_{1} \\
\hline
\end{array}
\end{aligned}
$$

An anonymous equivalence class (AEC) is the set of preference profiles that can be generated from each other via permuting the names of the voters, i.e. permuting the columns. That is, an AEC contains those preference profiles which are "equivalent" to each other if the names of the voters are immaterial. It can be shown (see Feller [5]) that the number of AECs for totally (linearly) ordered $m$ alternatives by $n$ voters is given by the binomial coefficient

$$
\left(\begin{array}{c}
n+m !-1 \\
m !-1
\end{array}\right)
$$

For this example there are four AECs: $\mathrm{AEC}_{1}=\left\{P_{1}\right\}, \mathrm{AEC}_{2}=\left\{P_{2}, P_{3}, P_{4}\right\}, \mathrm{AEC}_{3}=\left\{P_{5}, P_{6}, P_{7}\right\}$ and $\mathrm{AEC}_{4}=\left\{P_{8}\right\}$.

An anonymous and neutral equivalence class (ANEC) is the set of AECs that yield those preference profiles which are equivalent to each other if, not only the names of the voters, but also the names of the alternatives are immaterial. Hence, an ANEC contains those preference profiles that can be generated from each other via permuting the names of the voters and simultaneously the names of the alternatives. In the above example, there are two possible permutations for the names of the alternatives: one is the identity permutation which leaves the names of the alternatives 
intact, and the other is the permutation which re-labels $a_{1}$ as $a_{2}$ and $a_{2}$ as $a_{1}$. If we apply these permutations to the AECs mentioned above, we obtain a further partition of the set $\left\{\mathrm{AEC}_{1}, \mathrm{AEC}_{2}\right.$, $\left.\mathrm{AEC}_{3}, \mathrm{AEC}_{4}\right\}$ of anonymous equivalence classes. Note that this new partition gives us only two ANECs:

$$
\mathrm{ANEC}_{1}=\left\{\mathrm{AEC}_{1}, \mathrm{AEC}_{4}\right\}, \mathrm{ANEC}_{2}=\left\{\mathrm{AEC}_{2}, \mathrm{AEC}_{3}\right\}
$$

A representative preference profile in an ANEC is referred to as a root. A root representing ANEC $_{1}$ shows a preference structure at which all voters have the same preference ranking, and a root representing $\mathrm{ANEC}_{2}$ exhibits a structure where one of the preference rankings is adopted by two voters and the other is adopted by one voter.

Although the immediate way to compute the number of ANECs (roots) for a given $m$ and $n$ seems to be dividing the number of AECs by $m$ !, there are cases for which this does not work. Let us demonstrate the problem via the following example: For $m=2$ and $n=2$, there are four preference profiles:

$$
P_{1}=\begin{array}{|l|l|}
\hline a_{1} & a_{1} \\
\hline a_{2} & a_{2} \\
\hline
\end{array} \quad P_{2}=\begin{array}{|l|l|}
\hline a_{1} & a_{2} \\
\hline a_{2} & a_{1} \\
\hline
\end{array} \quad P_{3}=\begin{array}{|l|l|}
\hline a_{2} & a_{1} \\
\hline a_{1} & a_{2} \\
\hline
\end{array} \quad P_{4}=\begin{array}{|l|l|}
\hline a_{2} & a_{2} \\
\hline a_{1} & a_{1} \\
\hline
\end{array}
$$

The number of AECs is three: $\mathrm{AEC}_{1}=\left\{P_{1}\right\}, \mathrm{AEC}_{2}=\left\{P_{2}, P_{3}\right\}, \mathrm{AEC}_{3}=\left\{P_{4}\right\}$. Note that there are again two ANECs:

$$
\mathrm{ANEC}_{1}=\left\{\mathrm{AEC}_{1}, \mathrm{AEC}_{3}\right\}, \mathrm{ANEC}_{2}=\left\{\mathrm{AEC}_{2}\right\},
$$

and in this case $3 / 2$ ! is not even an integer. For $\mathrm{ANEC}_{2}$, both permutations of alternatives do not lead to anything other than $\mathrm{ANEC}_{2}$ itself, so it has only one AEC. Hence, an ANEC might contain less than $m$ ! AECs. We give in Theorem 11 a formula for the calculation of the number of ANECs, which explains the effect of the numerical properties of $n$ and $m$ in determining this number. A particular case is a result of Giritligil and Doğan [9] that, for a given pair of $m$ and $n$, each root represents $m$ ! AECs if and only if $m$ ! and $n$ are relatively prime.

\subsection{Probability models for sampling electorate's preferences: IC, IAC and IANC}

There are two widely adapted probability models in the social choice literature which are used for sampling voters' preferences. The IC model has been introduced by Guilbaud [11]. For totally ordered $m$ alternatives chosen by $n$ voters, IC assumes that each voter independently selects her preference ranking according to a uniform probability distribution. IC uses preference profiles for generating voters' preferences. This is a multinomial equiprobable preference profiles model which assumes that each of the $m !^{n}$ preference profiles is equally likely.

As introduced by Fishburn and Gehrlein [6], the IAC model relies also on an equiprobability assumption, but this time without taking the identity of the voters into account. Hence, the preferences of the electorate are generated via using anonymous profiles in which the names of the voters 
are neglected. An anonymous profile here is the representative profile selected from an anonymous equivalence class (AEC). IAC assumes that each anonymous profile is equally likely.

IC and IAC reflect the notions of Maxwell-Boltzmann and Bose-Einstein statistics, respectively, that are used in thermodynamics. The details about these assumptions and their extensive use in the literature are presented in Berg and Lepelley [1] and Gehrlein [7].

The impartial, anonymous and neutral culture (IANC) probability model of public choice theory that is introduced in this paper uses roots to generate voters' preferences. The very immediate reason for this approach is the fact that, all preference profiles and anonymous profiles that can be generated from a root via permuting the names of the alternatives and simultaneously the names of the voters have the same properties from the perspective of an anonymous and neutral SCR. For instance, if an anonymous and neutral SCR is detected to fail in satisfying certain criteria, then all of the preference profiles represented by this root suffer from the same problem. However, there is no reason for the other roots to have the paradox. That is, while the behavior of any such SCR is surely homogeneous among the profiles represented by any one root, it is not so across the roots. All roots that can be generated for a given pair of $m$ and $n$ form the set of all possible "preference structures" which are independent of voter and alternative names.

The number of preference profiles or anonymous profiles get tremendously large even for small numbers of alternatives (as small as five) and of voters. As a consequence, enumeration studies adopting IC or IAC fail to make exact calculations and clearly analyze the pattern of the behaviors of SCRs with respect to increasing number of alternatives and of voters. Apparently, the number of roots is small compared to the total number of preference profiles or anonymous profiles that can be generated for a given pair of $m$ and $n$. However, as presented in the preceding sections, the calculation of the number of roots is a non-trivial process due to the combinatorial complications induced by simultaneous permutation of the names of the alternative and of the voters.

We use elements of group theory to define ANECs and apply the Frobenius Lemma for the calculation of the number of roots. However, even then, we are faced with a combinatorial explosion in the calculation of the number of roots for large values of $m$ and $n$. At this point, we make use of certain properties of symmetric functions. We first develop a general formula for the number of roots, and then use this information along with the Dixon-Wilf algorithm to generate roots from a uniform distribution. We emphasize that a plain enumeration of the roots to this end is quite impossible to carry out.

Most importantly, it should be noted that the group theoretic approach and the application of certain symmetric function identities coupled with the Dixon-Wilf algorithm introduced in this paper can be adopted for different ways of generating voters' preferences. That is, the general framework of the presented model enables the researcher to work with preference profiles, anonymous profiles or roots to generate voters' preferences, and to analyze the behavior patterns of the SCRs for even high values of $m$ and $n$ (surely with modest hardware facilities) by Monte Carlo methods.

Some features of IANC that separates it further from IC and IAC are presented in the following sections. As applications of the method, the results of two Monte-Carlo simulations are given and 
compared with the ones of IC and IAC.

\section{Framework and formulation of roots}

\subsection{Permutations, partitions, and group actions}

We start by giving a brief outline of the elements of permutations, integer partitions, and group actions on finite sets and their application to the notion of roots. Our presentation is limited to the introduction of sufficient notation to be able to state the mathematical results that we present. For the proofs of the major results quoted, the reader is referred to [3].

Let $\Omega(m, n)$ denote the set of all preference profiles that can be generated for $m$ alternatives and $n$ voters. Note that the cardinality of $\Omega(m, n)$ is $m !^{n}$.

For the finite set of $n$ voters $[n]=\{1,2, \ldots, n\}$, a permutation $\sigma$ of $[n]$ is any one-to-one mapping of $[n]$ onto itself. Such a $\sigma$ can be regarded as a reordering of an ordered collection of $n$ distinct elements of $[n]$. The way that the elements of $[n]$ are reordered can be presented by a cycle decomposition of $\sigma$. For $n=3$, for example, the cycle decomposition of $\sigma=(12)(3)$ means that $\sigma$ takes the first element in the order to the second and the second element to the first, and fixes the third element (i.e. sends the third element to itself.)

The set of all permutations on $[n]$ form a group under the composition operation $\circ$, with the identity function from $[n]$ to $[n]$ forming the group identity. This group is called the symmetric group on $n$ symbols and denoted by $S_{n}$. The order of a group is the number of elements it contains. The order of $S_{n}$ equals $n$ !. For example, $S_{3}$ is of order six since it consists of six permutations which can be presented via the following cycle decompositions of $\sigma$ : (1)(2)(3), (12)(3), (1)(23), (13)(2), (123), (132).

A partition $\lambda$ of an integer $n$ is a weakly decreasing sequence of nonnegative integers $\lambda=\left(\lambda_{1} \geq\right.$ $\lambda_{2} \geq \cdots \geq \lambda_{n}$ ) with $n=\lambda_{1}+\lambda_{2}+\cdots+\lambda_{n}$. Each of the integers $\lambda_{i}>0$ is called a part of $\lambda$. For example $\lambda=(3,2,2)$ is a partition of $n=7$ into three parts. It has two parts of size two and one part of size three. If $\lambda$ is a partition of $n$, then this is denoted by $\lambda \vdash n$. Each partition of $n$ has a type denoted by the symbol $1^{\alpha_{1}} 2^{\alpha_{2}} \cdots n^{\alpha_{n}}$, which signifies that $\lambda$ has $\alpha_{i}$ parts of size $i$ for $1 \leq i \leq n$. For example the type of $\lambda=(3,2,2)$ is $1^{0} 2^{2} 3^{1} 4^{0} 5^{0} 6^{0} 7^{0}$. We can safely omit the zeros that appear as exponents and write the type of $\lambda$ as $2^{2} 3^{1}$.

A permutation $\sigma$ of $[n]$ defines a partition of $n$ where the parts of the partition are the cycle lengths in the cycle decomposition of $\sigma$. The cycle type of $\sigma$ is defined as the type of the resulting partition. For example $\sigma=(142)(35)(67)$ has cycle type $2^{2} 3^{1}$. For any $\lambda \vdash n$, define the number

$$
z_{\lambda}=1^{\alpha_{1}} 2^{\alpha_{2}} \cdots n^{\alpha_{n}} \alpha_{1} ! \alpha_{2} ! \cdots \alpha_{n} ! .
$$

It is well known that the number of permutations of cycle type $1^{\alpha_{1}} 2^{\alpha_{2}} \cdots n^{\alpha_{n}}$ is given by $z_{\lambda}^{-1} n$ ! where $\lambda$ is the partition of cycle lengths of $\sigma$. For example in the symmetric group $S_{7}$, there are

$$
\frac{7 !}{2^{2} 3^{1} 2 ! 1 !}=210
$$


permutations having the same cycle type $2^{2} 3^{1}$ as $\sigma$. The collection of permutations which have a given cycle type is called a conjugacy class.

A group of permutations $G$ acts on a set $\Omega$ if each element $\sigma$ of the group permutes the elements of $\Omega$, in such a way that the identity element does nothing, while a composition of actions corresponds to the action of the composition. Denote by $P^{\sigma}$ the image of $P \in \Omega$ under the permutation of $\Omega$ induced by $\sigma$. Then, for $P \in \Omega$ and $\sigma, \tau \in G,\left(P^{\sigma}\right)^{\tau}=P^{\tau \circ \sigma}$. The subset $\left\{P^{\sigma} \mid \sigma \in G\right\}$ of $\Omega$ is called the group orbit of $P \in \Omega$. A group action on $\Omega$ splits up $\Omega$ into a disjoint union of subsets

$$
\Omega=\theta_{1}+\theta_{2}+\cdots+\theta_{t}
$$

where each $\theta_{i}$ is a group orbit and the "+" signifies disjoint union. In fact the $\theta_{i}$ are the equivalence classes under the action of $G$ on $\Omega$ where we define $P, Q \in \Omega$ to be equivalent and put $P \sim Q$ if and only if there exists some permutation $\sigma \in G$ such that $Q=P^{\sigma}$. If $P^{\sigma}=P$ then $P$ is a fixed-point of $\sigma$. If we put $G_{P}=\left\{\sigma \in G \mid P^{\sigma}=P\right\}$, then this is called the stabilizer subgroup of $P \in \Omega$. $G_{P}$ is a group in its own right. As a consequence of the orbit-stabilizer theorem and Lagrange's theorem, the number of elements in the group orbit of $P$ is given by the quotient $|G| /\left|G_{P}\right|$. For $\sigma \in G$ let $F_{\sigma}=\left\{P \in \Omega \mid P^{\sigma}=P\right\}$ denote the set of elements of $\Omega$ fixed by $\sigma$. Consider now a group of permutations $G$ acting on a set $\Omega$. As in (5), this action splits up $\Omega$ into equivalence classes. The number $t$ of these classes can be computed by the formula

$$
t=\frac{1}{|G|} \sum_{\sigma \in G}\left|F_{\sigma}\right|
$$

which is known as the Frobenius lemma (or Burnside lemma). This lemma says that the number of orbits under the action of a finite group $G$ is the average number of fixed points of elements of $G$.

For detailed information on permutations groups and their actions on finite sets, we we refer the reader to Kerber [12], or Wielandt [15].

\subsection{The number of roots for $m$ alternatives and $n$ voters}

First we need some more definitions and notation. For integers $d$ and $n$ we use the symbol $d \mid n$ to mean that $d$ divides $n$ evenly. For any statement $S$ we define the indicator function of $S$ by

$$
\chi(S)= \begin{cases}1 & \text { if } S \text { is True, } \\ 0 & \text { if } S \text { is False } .\end{cases}
$$

For partitions $\lambda$ and $\mu$, we use $G C D(\lambda)$ as a shorthand for the greatest common divisor (GCD) of the parts of $\lambda$, and $\operatorname{LCM}(\mu)$ as a shorthand for the least common multiple (LCM) of the parts of $\mu$.

For an integer $k$ with $0 \leq k \leq x$, extend the definition of the ordinary binomial coefficient $\left(\begin{array}{l}x \\ k\end{array}\right)$ to nonintegral values of $x$ by setting

$$
\left(\begin{array}{l}
x \\
k
\end{array}\right)= \begin{cases}\frac{x !}{k !(x-k) !} & \text { if } x \text { is integral, } \\
0 & \text { otherwise. }\end{cases}
$$

We remark that this extension is distinct from the standard one which makes use of the $\Gamma$ function to extend the factorials. 
Given $n$ and $m$, let $\Omega=\Omega(m, n)$ consist of all $m \times n$ matrices with entries from $\left\{a_{1}, a_{2}, \ldots, a_{m}\right\}$ such that each of the $n$ columns is a permutation of $\left\{a_{1}, a_{2}, \ldots, a_{m}\right\}$. The product group $S_{n} \times S_{m}$ acts on $\Omega$. $S_{n} \times S_{m}$ consists of pairs $g=(\sigma, \tau)$ with $\sigma \in S_{n}$ and $\tau \in S_{m}$, where the group operation is componentwise composition of permutations. In the action of $g=(\sigma, \tau)$, a preference profile $P^{g}$ is obtained from another preference profile $P$ by permuting the columns (voters) according to $\sigma$, and simultaneously permuting the alternative names in $P$ by mapping each $a_{i}$ to $a_{\tau(i)}, i=1,2, \ldots, m$. Consider the preference profile $P$ below for $m=2$ and $n=4$.

$$
P=\begin{array}{|l|l|l|l|}
\hline a_{1} & a_{1} & a_{2} & a_{2} \\
\hline a_{2} & a_{2} & a_{1} & a_{1} \\
\hline
\end{array}
$$

If we take $g=((13)(24),(1)(2))$, then

$$
P^{g}=\begin{array}{|l|l|l|l|}
\hline a_{2} & a_{2} & a_{1} & a_{1} \\
\hline a_{1} & a_{1} & a_{2} & a_{2} \\
\hline
\end{array}
$$

and for $g=((13)(24),(12))$, we have

$$
P^{g}=\begin{array}{|l|l|l|l|}
\hline a_{1} & a_{1} & a_{2} & a_{2} \\
\hline a_{2} & a_{2} & a_{1} & a_{1} \\
\hline
\end{array}=P .
$$

The number of roots $R(m, n)$ is given by the number of group orbits $t$ in the decomposition (5).

Theorem 1 The number of roots $R(m, n)$ is given by

$$
R(m, n)=\sum_{\substack{\lambda \vdash n \\ \mu \vdash m}} \chi(L C M(\mu) \mid G C D(\lambda)) z_{\mu}^{-1} z_{\lambda}^{-1} m !^{\alpha_{1}+\cdots+\alpha_{n}}
$$

where the type of $\lambda$ is $1^{\alpha_{1}} 2^{\alpha_{2}} \cdots n^{\alpha_{n}}$ and $z_{\lambda}$ is as defined in (4).

We apply the Frobenius lemma and determine the nature of the fixed points of $g \in S_{n} \times S_{m}$. The proofs of the main Theorems 1, 2, and 3 and the elements of the theory of symmetric functions that we make use of can be found in [3]. We state these results here to convince the reader that there is a rigorous basis to the algorithm we have produced for the generation of roots with equal probability.

The expression on the right-hand side of $(8)$ for $R(m, n)$ is actually a double sum, and the number of terms involved in the summation is the product of the number of partitions of $n$ and the number of partitions of $m$. Since the number of partitions of an integer grows exponentially, the evaluation of $R(m, n)$ via (8) is not practical.

Now suppose that $\operatorname{LCM}(\mu)=d$. Then the contribution of $\mu$ to the sum (8) can be written as

$$
\begin{aligned}
& z_{\mu}^{-1} \sum_{\lambda \vdash n} z_{\lambda}^{-1} m !^{\alpha_{1}+\cdots+\alpha_{n}} \\
& d \mid \lambda_{i}, \forall i
\end{aligned}
$$


To be able to use this to simplify the number of terms in the computation of $R(m, n)$, we need a simpler expression for this sum. Fortunately, a formula for (9) can be found by using methods from the theory of symmetric functions (see Macdonald [14], [3]).

Theorem 2 For any two integers $n$ and $r$

$$
\sum_{\substack{\lambda \vdash n \\
d \mid \lambda_{i}, \forall i}} z_{\lambda}^{-1} r^{\alpha_{1}+\cdots+\alpha_{n}}=\left(\begin{array}{c}
\frac{n}{d}+\frac{r}{d}-1 \\
\frac{r}{d}-1
\end{array}\right)
$$

where the binomial coefficient is defined as in (7).

These two results together yield

\section{Theorem 3}

$$
R(m, n)=\sum_{\mu \vdash m} z_{\mu}^{-1}\left(\begin{array}{c}
\frac{n}{d}+\frac{m !}{d}-1 \\
\frac{m !}{d}-1
\end{array}\right)
$$

where $d=d(\mu)=L C M(\mu)$, the binomial coefficient is defined as in (7), and $z_{\mu}$ is as defined in (4).

Note that the summation in (11) is over partitions of $m$ only, and is independent of the number of voters $n$. Theorem 3 has some immediate implications. We take a look at some simple examples and applications.

Formula for $n$ voters and $m=2$ alternatives

For $m=2$, there are only two partitions of $m$. These are $(1,1)$ and $(2)$. We have $\operatorname{LCM}(1,1)=1$, and $\operatorname{LCM}(2)=2$. The corresponding values of $z_{\mu}$ are both 2 . Thus we obtain an exact formula for the number of roots as

$$
\begin{aligned}
R(2, n) & =\frac{1}{2} \sum_{\lambda \vdash n} z_{\lambda}^{-1} 2 !^{\alpha_{1}+\cdots+\alpha_{n}}+\frac{1}{2} \sum_{\substack{\lambda \vdash n \\
2 \mid \lambda_{i}}} z_{\lambda}^{-1} 2 !^{\alpha_{1}+\cdots+\alpha_{n}} \\
& =\frac{1}{2}\left(\begin{array}{c}
n+1 \\
1
\end{array}\right)+\frac{1}{2}\left(\begin{array}{c}
\frac{n}{2} \\
0
\end{array}\right)
\end{aligned}
$$

This is another way of saying

$$
R(2, n)= \begin{cases}\frac{1}{2} n+1 & \text { if } n \text { is even } \\ \frac{1}{2}(n+1) & \text { if } n \text { is odd }\end{cases}
$$

Example: For the example with $m=2$ and $n=3$ given in in Section $2.1, R(2,3)=2$ orbits (roots) of $m !^{n}=8$ elements of $\Omega(2,3)$ are the two given in (2) as $\mathrm{ANEC}_{1}$ and $\mathrm{ANEC}_{2}$.

Example: For the example with $m=2$ and $n=2$ given in Section $2.1, R(2,2)=2$ orbits (roots) of $m !^{n}=4$ elements of $\Omega(2,2)$ are the two given in $(3)$ as $\mathrm{ANEC}_{1}$ and $\mathrm{ANEC}_{2}$. 
Example: $\quad$ For $m=2$ and $n=4$, the $R(2,4)=3$ orbits (roots) of $m !^{n}=16$ elements of $\Omega(2,4)$ are given by

$$
\begin{aligned}
& \theta_{1}=\left\{\begin{array}{|l|l|l|l|}
\hline a_{1} & a_{1} & a_{1} & a_{1} \\
\hline a_{2} & a_{2} & a_{2} & a_{2} \\
\hline
\end{array}, \begin{array}{|l|l|l|l|}
\hline a_{2} & a_{2} & a_{2} & a_{2} \\
\hline a_{1} & a_{1} & a_{1} & a_{1} \\
\hline
\end{array}\right\} \\
& \theta_{2}=\left\{\begin{array}{|l|l|l|l}
\hline a_{1} & a_{1} & a_{2} & a_{2} \\
\hline a_{2} & a_{2} & a_{1} & a_{1} \\
\hline
\end{array}, \begin{array}{|l|l|l|l|}
\hline a_{1} & a_{2} & a_{1} & a_{2} \\
\hline a_{2} & a_{1} & a_{2} & a_{1} \\
\hline
\end{array}, \begin{array}{|l|l|l|l|}
\hline a_{2} & a_{2} & a_{1} & a_{1} \\
\hline a_{1} & a_{1} & a_{2} & a_{2} \\
\hline
\end{array}, \begin{array}{|l|l|l|l|}
a_{2} & a_{1} & a_{2} & a_{1} \\
\hline a_{1} & a_{2} & a_{1} & a_{2} \\
\hline
\end{array},\right. \\
& \left.\begin{array}{|l|l|l|l|}
\hline a_{1} & a_{2} & a_{2} & a_{1} \\
\hline a_{2} & a_{1} & a_{1} & a_{2} \\
\hline
\end{array}, \begin{array}{|l|l|l|l|}
\hline a_{2} & a_{1} & a_{1} & a_{2} \\
\hline a_{1} & a_{2} & a_{2} & a_{1} \\
\hline
\end{array}\right\} \\
& \theta_{3}=\left\{\begin{array}{|l|l|l|l}
\hline a_{1} & a_{1} & a_{1} & a_{2} \\
\hline a_{2} & a_{2} & a_{2} & a_{1} \\
\hline
\end{array}, \begin{array}{|l|l|l|l}
\hline a_{1} & a_{1} & a_{2} & a_{1} \\
\hline a_{2} & a_{2} & a_{1} & a_{2} \\
\hline
\end{array}, \begin{array}{|l|l|l|l|}
\hline a_{1} & a_{2} & a_{1} & a_{1} \\
\hline a_{2} & a_{1} & a_{2} & a_{2} \\
\hline
\end{array}, \begin{array}{|l|l|l|l|}
a_{2} & a_{1} & a_{1} & a_{1} \\
\hline a_{1} & a_{2} & a_{2} & a_{2} \\
\hline
\end{array},\right. \\
& \left.\begin{array}{|l|l|l|l}
\hline a_{1} & a_{2} & a_{2} & a_{2} \\
\hline a_{2} & a_{1} & a_{1} & a_{1} \\
\hline
\end{array}, \begin{array}{|l|l|l|l|}
\hline a_{2} & a_{1} & a_{2} & a_{2} \\
\hline a_{1} & a_{2} & a_{1} & a_{1} \\
\hline a_{1} & a_{1} & a_{2} & a_{1} \\
\hline a_{2} & a_{2} & a_{1} & a_{2} \\
\hline a_{1} & a_{1} & a_{1} & a_{2} \\
\hline a_{2} & a_{2} & a_{2} & a_{1} \\
\hline
\end{array}\right\}
\end{aligned}
$$

\section{Formula for $n$ voters and $m=3$ alternatives}

For $m=3$, there are a total of three partitions of $m$. These are $(1,1,1),(2,1)$, and $(3)$. We have $\operatorname{LCM}(1,1,1)=1, \operatorname{LCM}(2,1)=2$, and $\operatorname{LCM}(3)=3$. The corresponding values of $z_{\mu}$ are 6,2 , and 3 , respectively. Thus we obtain an exact formula for the number of roots as

$$
\begin{aligned}
R(3, n)= & \frac{1}{6} \sum_{\lambda \vdash n} z_{\lambda}^{-1} 3 !^{\alpha_{1}+\cdots+\alpha_{n}}+\frac{1}{2} \sum_{\lambda \vdash n} z_{\lambda}^{-1} 3 !^{\alpha_{1}+\cdots+\alpha_{n}}+\frac{1}{3} \sum_{\lambda \vdash n} z_{\lambda}^{-1} 3 !^{\alpha_{1}+\cdots+\alpha_{n}} \\
& =\frac{1}{6}\left(\begin{array}{c}
n+5 \\
5
\end{array}\right)+\frac{1}{2}\left(\begin{array}{c}
\frac{n}{2}+2 \\
2
\end{array}\right)+\frac{1}{3}\left(\begin{array}{c}
\frac{n}{3}+1 \\
1
\end{array}\right)
\end{aligned}
$$

When $n$ and $m$ ! are relatively prime, only one of the terms involving the extended binomial coefficients is nonzero, and the summation in Theorem 3 has a single term corresponding to $d=1$ only. The corresponding partition is $\mu=(1,1, \ldots, 1)$ with $z_{\mu}=m$ !. An immediate corollary is the following result mentioned in the introduction:

Corollary When $n$ and $m$ ! are relatively prime, the number of roots $R(m, n)$ is given by

$$
R(m, n)=\frac{1}{m !}\left(\begin{array}{c}
n+m !-1 \\
m !-1
\end{array}\right)
$$

\section{A comparison of the number of AECs and roots for $m=3$ alternatives}

For $m=3$ alternatives, the number of roots is given by the sum in (13). The number of AECs is given by the binomial coefficient in (1). Figure 1 compares these numbers for $1 \leq n \leq 100$, where the vertical axis is log-scale.

Counting the roots by means of the formulas given in Theorems 1, 2 and 3 may seem unwieldy in general, since as we have remarked the number of partitions of $n$ grows exponentially in $n$. However, 


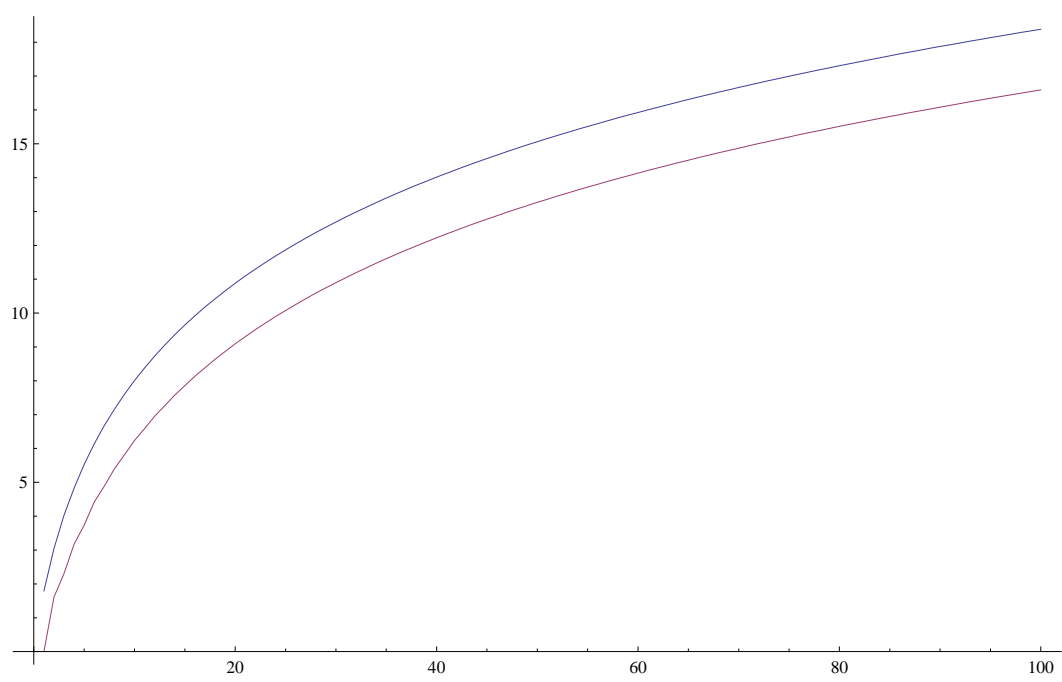

Figure 1: The number of AECs and ANECs(roots). The larger value plotted is the number of AECs, and the smaller one is the number of roots. The number of voters $n$ ranges from 1 to 100 . The vertical axis is given in the log-scale.

the calculation of $R(m, n)$ by means of a symbolic algebra package such as Mathematica is relatively straightforward. We can easily calculate the value of $R(m, n)$ for relatively large values of $m$ and $n$ using the general formula in Theorem 3 . As examples

$$
\begin{aligned}
R(5,5) & =1876255 \\
R(5,10) & =2049242056940 \\
R(5,20) & =5908312923863263889174 \\
R(5,30) & =214658568936630826879925768420
\end{aligned}
$$

as well as incomprehensibly large values such as

17758069318650119858962669239674956879327271587948512064321599292646589480794890970501606240176140489486440

for $R(10,20)$.

In the next section, we shall indicate the importance of being able to access the values of $R(m, n)$ for large and unconstrained values of $m$ and $n$. The ability to compute the value of $R(m, n)$ together with a general combinatorial method known as the Dixon-Wilf algorithm allows us to construct a symbolic package to generate the roots such that each root is produced with probability $1 / R(m, n)$.

Next we describe the ingredients we use in the construction of our symbolic algebra package.

\section{A Monte-Carlo testbed for IANC}

Our immediate aim in the construction of this testbed is to be able to generate representatives of the roots randomly where each root is equally likely. Fortunately, the theory of group actions on 
sets that we have used for the interpretation of the roots as the orbits of a group action allows us to do this. In addition to having access to the elements of this procedure, our group is an explicit permutation group $S_{n} \times S_{m}$, and we are also able to calculate the quantities, such as the size of the conjugacy classes, that are needed in the standard theory of symmetric functions.

\subsection{Uniform generation of roots}

Suppose a group of permutations $G$ acts on a set $\Omega$. Consider the decomposition of $\Omega$ into orbits $\theta_{1}, \theta_{2}, \ldots, \theta_{t}$ as in (5). If the number of orbits $t$ is known (this is $R(m, n)$ in our case), then the following procedure, usually referred to as the Dixon-Wilf algorithm in the combinatorics literature (Dixon and Wilf [2]) can be used to generate an orbit $\theta$ from the uniform distribution.

\section{Basic elements of the Dixon-Wilf Algorithm}

1. Select a conjugacy class $C \subseteq G$ with probability $\frac{|C|\left|F_{\sigma}\right|}{t|G|}$, where $\sigma$ is some member of $C$.

2. Select uniformly at random some $x \in F_{\sigma}$.

3. Return the orbit $\theta$ that contains $x$.

The crucial aspect of the Dixon-Wilf algorithm is that it is guaranteed to return an orbit (representative) distributed uniformly over the set of all orbits.

Recall again the example with $m=2$ and $n=3$ in Section 2.1. In this case, we have $\left|\theta_{1}\right|=2$ and $\left|\theta_{2}\right|=6$. If each of the 8 preference profiles were equally likely, then that would generate an element from $\theta_{1}$ with probability $1 / 4$ and from $\theta_{2}$ with probability $3 / 4$. In contrast, the Dixon-Wilf algorithm guarantees that an element from $\theta_{1}$ or an element from $\theta_{2}$ will be returned, each case being equally likely. In this case each $P \in \theta_{1}$ is returned with probability $1 / 4$, and each $P \in \theta_{2}$ is returned with probability $1 / 12$, although the values of what these individual probabilities are need not concern us. What is important is that each root is an equally likely outcome.

By means of Theorems 1, 2 and 3, we are able calculate the number of orbits $t=R(m, n)$ for large values of $m$ and $n$. In addition, we can calculate the necessary parameters as required in the Dixon-Wilf algorithm such as the size of the conjugacy classes for the product group of IANC. The details can be found in [3].

We have implemented an algorithm named GenerateRoot $[\mathrm{m}, \mathrm{n}]$ to generate roots from the uniform distribution as a Mathematica program (the Mathematica notebook containing this function can be online for experimentation: see [4]). The program takes two integers $m, n$ as input and generates a preference profile $P$ by our algorithm, which is based on the Dixon-Wilf algorithm. The resulting $P$ is guaranteed to be distributed over the $R(m, n)$ roots uniformly. This is the surprising application of the Dixon-Wilf algorithm.

As an example of the performance of the algorithm, Table 4.1 gives the numerical results from runs of the root generation algorithm for a special case. We have run the algorithm $x$ times for $x$ running from 10 to 10000 in powers of 10 for $m=2$ and $n=4$. For each preference profile $P$ 
returned by GenerateRoot $[2,4]$ we checked whether $P \in \theta_{1}, P \in \theta_{2}$, or $P \in \theta_{3}$. These are the three roots for $m=2$ and $n=4$ as given in (12). $\operatorname{Pr}$ [Hits from orbit $\theta_{1}$ ] is the ratio of the number of $P \in \theta_{1}$ to $x$. $\operatorname{Pr}\left[\right.$ Hits from orbit $\left.\theta_{2}\right]$ and $\operatorname{Pr}\left[\right.$ Hits from orbit $\left.\theta_{3}\right]$ are calculated similarly. The resulting probabilities are collected in Table 4.1. Since there are 3 roots, the actual probability for each is $0.333 \ldots$

\begin{tabular}{|l|l|l|l|}
\hline No. of trials $x$ & $\operatorname{Pr}\left[\right.$ Hits from orbit $\left.\theta_{1}\right]$ & $\operatorname{Pr}\left[\right.$ Hits from orbit $\left.\theta_{2}\right]$ & $\operatorname{Pr}\left[\right.$ Hits from orbit $\left.\theta_{3}\right]$ \\
\hline 10 & 0.2 & 0.5 & 0.3 \\
\hline 100 & 0.34 & 0.29 & 0.37 \\
\hline 1000 & 0.352 & 0.348 & 0.3 \\
\hline 10000 & 0.3235 & 0.3396 & 0.3369 \\
\hline
\end{tabular}

Table 1: Random generation of roots from the uniform distribution with 4 voters and 2 alternatives. Each trial is the generation of a root from $R(2,4)$ by using GenerateRoot $[2,4]$. The three roots $\theta_{1}, \theta_{2}, \theta_{3}$ area as given in (12).

\subsection{Variations on the IANC model, homogeneity}

We have considered $m \times n$ matrices $\Omega=\Omega(m, n)$ with entries from the alternatives $\left\{a_{1}, a_{2}, \ldots, a_{m}\right\}$ such that each of the $n$ columns is a permutation of $\left\{a_{1}, a_{2}, \ldots, a_{m}\right\}$. This is the collection of $m \times n$ preference profiles. For our model, the product group $S_{n} \times S_{m}$ acts on $\Omega$, and the roots are representatives of the equivalence classes under this action. The more common models have also group theoretic interpretation in which only the group is different. For example, IC corresponds to the action by the trivial group $e \times e$ which fixes every profile in $\Omega$. The IAC is the equivalence classes of the action of $S_{n}$ only in the form $S_{n} \times e$, where the names of the alternatives are left fixed. We can summarize the situation in Figure 4.2.

\begin{tabular}{|c|c|c|}
\hline Group action & Voter preference model & Number of inequivalent classes of profiles (roots) \\
\hline$e$ & IC & $m !^{n}$ \\
\hline$S_{n}$ & IAC & $\left(\begin{array}{c}n+m !-1 \\
m !-1\end{array}\right)$ \\
\hline$S_{n} \times S_{m}$ & IANC & $R(m, n)$ \\
\hline
\end{tabular}

Table 2: Group actions on preference profiles with $m$ alternatives and $n$ voters which give the IC, IAC, and IANC models. The formula for $R(m, n)$ is as given in Theorem 3.

Group theoretic formulation and the resulting Monte-Carlo generation tools can be useful in the analyses of other types of models as well. For example, suppose that the alternatives come from two parties, say a subset $R$ of red alternatives, and a complementary subset $B$ of blue alternatives. We can then impose the neutrality condition that the red alternatives are equivalent under the relabelings of $R$, and the blue alternatives are equivalent under the relabelings of $B$. If the number 
of alternatives in $R$ and $B$ are $r$ and $b=m-r$, then we have a natural action of the group $S_{n} \times S_{r} \times S_{b}$ on the preference profiles with $n$ voters and $m$ alternatives. Therefore many structural questions in social choice theory can be formulated in a setting of this type and then analyzed by Monte-Carlo methods.

Both IC and IAC have been characterized in terms of special Pólya-Eggenberger urn models with $m$ ! balls that correspond to the possible total orders of the alternatives. The probabilities can then be described by taking the parameter $\alpha=0$ for the IC and and $\alpha=1$ for IAC. A recent exposition of these calculations can be found in Lepelley and Valognes [13]. The parameter $\alpha$ can be interpreted as a homogeneity measure for the voters. In the case $\alpha=0$ the urn model is without replacement and the voters form their opinion independently of one another. For positive $\alpha$, in particular for IAC where $\alpha=1$, the urn model is now with replacement and the homogeneity interpretation is that a certain amount of dependence exists between the preferences of different voters.

For the IANC, it is unlikely that there is a similar Pólya-Eggenberger urn model that allows for the the homogeneity interpretation for the model. The difficulty is in the nature of the balls, since for the IANC the difference is not only in the replacement rule but also the stipulated equality of some of the balls after they have been picked. However, since the equivalence classes of IANC are coarser than the equivalence classes of IAC, we can safely say that there is at least as much dependence between the preferences of different voters as there are in the IAC model.

\section{Applications: Monte-Carlo simulations}

We demonstrate the applications of IANC by providing two Monte-Carlo experiments. For these applications we focus on two anonymous and neutral SCRs that are known and studied the most in the literature: Plurality and the Condorcet rule.

\subsection{Plurality and the Condorcet rule}

How to aggregate individual preferences into a social choice has been a major ethical question ever since the political philosophy of the Enlightenment. When only two alternatives are at stake, the ordinary majority voting is unambiguously regarded as the "fairest" method. For three and more alternatives, plurality voting, where each voter reports the name of exactly one alternative on her ballot and the alternative receiving the most votes wins, has been historically the most popular SCR.

One of the celebrated critiques of plurality voting, Marquis de Condorcet [17] noted that plurality voting may elect a poor candidate, namely, it can result in a winner that would lose in a simple pair-wise majority comparison to every other candidate. Condorcet provided the most analyzed non-positionalist voting principle by requiring that if a candidate defeats every other candidate on the basis of the simple majority rule, then that candidate should be the winner in the election.

Example: Consider the preference profile for $m=4$ alternatives $A=\{a, b, c, d\}$ and $n=5$ voters 
given below:

\begin{tabular}{|l|l|l|l|l|}
\hline$a$ & $a$ & $b$ & $c$ & $d$ \\
\hline$b$ & $b$ & $c$ & $d$ & $c$ \\
\hline$c$ & $d$ & $d$ & $b$ & $b$ \\
\hline$d$ & $c$ & $a$ & $a$ & $a$ \\
\hline
\end{tabular}

In the above example, the alternative $a$ is the plurality winner since it is top-ranked the most among all available alternatives. However, note that it is the very last choice of the majority (3 out of 5 ) of voters. In order to be able to determine the Condorcet winner in this example, we need to check the pairwise majority relations between the alternatives: $b$ beats $a, c$ and $d$ in 3 out of 5 voters' rankings. Therefore, $b$ is the Condorcet winner. Note that $a$, the plurality winner, is beaten by every other alternative and it is actually the Condorcet loser.

The Condorcet rule has been widely studied not only as a SCR but also as a desirable criterion. However, this principle has also generated one of the most studied paradoxes in the social choice literature. Condorcet paradox occurs when a social outcome is cyclic (i.e, not transitive) even though the individual preferences are not, due to the conflict in majority wishes.

Example: Consider the preference profile for $m=3$ alternatives $A=\{a, b, c\}$ and $n=3$ voters given below:

\begin{tabular}{|l|l|l|}
\hline$a$ & $b$ & $c$ \\
\hline$b$ & $c$ & $a$ \\
\hline$c$ & $a$ & $b$ \\
\hline
\end{tabular}

In this profile $a$ is preferred to $b$ and $b$ is preferred to $c$, however, $c$ is preferred to $a$ in pairwise majority relation. That is, majority wishes are cyclic and there is no alternative which beats every other alternative in majority of the voter preferences. Hence, the Condorcet rule fails to choose a winner.

Based on the possibility of non-existence of a Condorcet winner, the criteria called Condorcet consistency of a SCR is defined as the conditional probability that the given SCR chooses the Condorcet winner, given that a Condorcet winner exists.

\subsection{Likelihood of the existence of a Condorcet winner}

Although certain domains of preferences that guarantee the existence of Condorcet winner are known theoretically (the so called single-peaked preferences), these do not fully characterize the preferences that have Condorcet winners. Since Condorcet rule serves also as a certain criterion of desirability, it is of interest to know the likelihood of existence of a Condorcet winner.

For $n=9$ and $m=6$, the two randomly generated preference profiles (i.e. roots) produced by GenerateRoot $[\mathrm{m}, \mathrm{n}]$ in Figure 2 both have Condorcet winners, whereas the randomly generated 
preference profile in Figure 3 does not have a Condorcet winner. Thus, if we had picked only three random roots using GenerateRoot [6,9], then 2 out of 3 generated have Condorcet winners. We may be tempted to extrapolate that maybe 2 out of 3 roots have Condorcet winners in the general case of 9 voters and 6 alternatives. GenerateRoot [6,9] allows us to carry this experiment much further. We have run the root generation algorithm $x$ times for $x$ running from 10 to 100000 in powers of 10 for $m=6$ and $n=9$. For each $P$ returned by GenerateRoot $[6,9]$ we checked whether the profile $P$ has a Condorcet winner. Considering the results in Figure 3, it appears that the probability of a Condorcet winner over all the roots is about 0.74 .

\begin{tabular}{|l|l|l|l|l|l|l|l|l|}
\hline$a_{2}$ & $a_{5}$ & $a_{3}$ & $a_{4}$ & $a_{3}$ & $a_{5}$ & $a_{2}$ & $a_{2}$ & $a_{2}$ \\
\hline$a_{6}$ & $a_{3}$ & $a_{4}$ & $a_{5}$ & $a_{6}$ & $a_{1}$ & $a_{1}$ & $a_{6}$ & $a_{6}$ \\
\hline$a_{5}$ & $a_{6}$ & $a_{2}$ & $a_{1}$ & $a_{1}$ & $a_{6}$ & $a_{6}$ & $a_{1}$ & $a_{1}$ \\
\hline$a_{3}$ & $a_{2}$ & $a_{5}$ & $a_{6}$ & $a_{4}$ & $a_{4}$ & $a_{5}$ & $a_{3}$ & $a_{3}$ \\
\hline$a_{4}$ & $a_{4}$ & $a_{1}$ & $a_{2}$ & $a_{2}$ & $a_{2}$ & $a_{3}$ & $a_{4}$ & $a_{4}$ \\
\hline$a_{1}$ & $a_{1}$ & $a_{6}$ & $a_{3}$ & $a_{5}$ & $a_{3}$ & $a_{4}$ & $a_{5}$ & $a_{5}$ \\
\hline
\end{tabular}

\begin{tabular}{|l|l|l|l|l|l|l|l|l|}
\hline$a_{1}$ & $a_{3}$ & $a_{6}$ & $a_{2}$ & $a_{5}$ & $a_{3}$ & $a_{1}$ & $a_{1}$ & $a_{2}$ \\
\hline$a_{4}$ & $a_{2}$ & $a_{2}$ & $a_{5}$ & $a_{2}$ & $a_{2}$ & $a_{4}$ & $a_{3}$ & $a_{4}$ \\
\hline$a_{5}$ & $a_{5}$ & $a_{4}$ & $a_{3}$ & $a_{6}$ & $a_{5}$ & $a_{5}$ & $a_{4}$ & $a_{6}$ \\
\hline$a_{2}$ & $a_{4}$ & $a_{3}$ & $a_{1}$ & $a_{1}$ & $a_{6}$ & $a_{6}$ & $a_{5}$ & $a_{5}$ \\
\hline$a_{6}$ & $a_{6}$ & $a_{5}$ & $a_{4}$ & $a_{4}$ & $a_{1}$ & $a_{2}$ & $a_{2}$ & $a_{3}$ \\
\hline$a_{3}$ & $a_{1}$ & $a_{1}$ & $a_{6}$ & $a_{3}$ & $a_{4}$ & $a_{3}$ & $a_{6}$ & $a_{1}$ \\
\hline
\end{tabular}

Figure 2: Two randomly generated preference profiles with 9 voters and 6 alternatives that happen to have Condorcet winners.

\begin{tabular}{|l|l|l|l|l|l|l|l|l|}
\hline$a_{5}$ & $a_{3}$ & $a_{4}$ & $a_{6}$ & $a_{3}$ & $a_{3}$ & $a_{4}$ & $a_{3}$ & $a_{6}$ \\
\hline$a_{4}$ & $a_{5}$ & $a_{1}$ & $a_{2}$ & $a_{5}$ & $a_{6}$ & $a_{5}$ & $a_{6}$ & $a_{5}$ \\
\hline$a_{6}$ & $a_{1}$ & $a_{5}$ & $a_{4}$ & $a_{1}$ & $a_{5}$ & $a_{2}$ & $a_{2}$ & $a_{4}$ \\
\hline$a_{2}$ & $a_{4}$ & $a_{3}$ & $a_{3}$ & $a_{2}$ & $a_{4}$ & $a_{6}$ & $a_{1}$ & $a_{3}$ \\
\hline$a_{3}$ & $a_{6}$ & $a_{2}$ & $a_{1}$ & $a_{4}$ & $a_{1}$ & $a_{3}$ & $a_{4}$ & $a_{1}$ \\
\hline$a_{1}$ & $a_{2}$ & $a_{6}$ & $a_{5}$ & $a_{6}$ & $a_{2}$ & $a_{1}$ & $a_{5}$ & $a_{2}$ \\
\hline
\end{tabular}

Figure 3: A randomly generated preference profile with 9 voters and 6 alternatives that does not have a Condorcet winner.

\begin{tabular}{|c|c|}
\hline No. of trials $x$ & $\operatorname{Pr}$ [Existence of a Condorcet winner] \\
\hline 10 & 0.8 \\
\hline 100 & 0.73 \\
\hline 1000 & 0.75 \\
\hline 10000 & 0.733 \\
\hline 100000 & 0.73705 \\
\hline
\end{tabular}

Table 3: For $n=9$ and $m=6$, the probability of roots to have a Condorcet winner in the IANC model.

Figure 4 shows the computed probabilities of the existence of Condorcet winners in the IANC 
model for $m=3$ alternatives, obtained by running GenerateRoot $[3, \mathrm{n}]$ for up to $n=41$ voters.

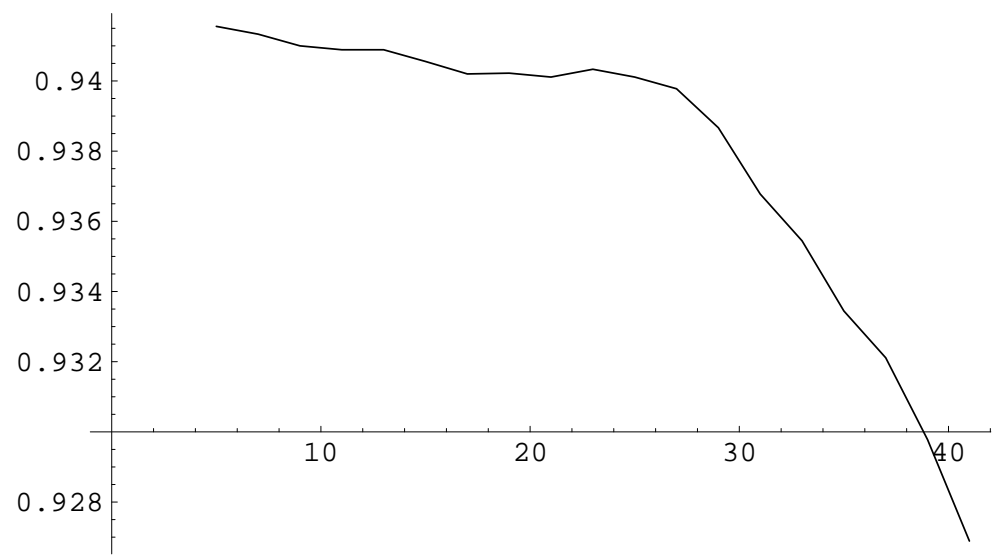

Figure 4: The probability of the existence of Condorcet winners for $m=3$ alternatives in the IANC model. Note the scale of the vertical axis and the small range of probabilities shown. The horizontal axis is the number of voters $n$, through odd integers from 5 to 41 . Data has been smoothed by a 5 -term moving-average filter. The number of samples used is $N=10000$.

\subsection{Condorcet consistency of Plurality}

In this section, we report the results of the Monte-Carlo simulations we have performed to experimentally determine the likelihood of plurality to choose the Condorcet winner. This is also called the Condorcet consistency of plurality. Since plurality is the most commonly used SCR due to the ease of its implementation in terms of the amount of information required from the voters, its fulfillment of the Condorcet criteria, i.e. its Condorcet consistency is important.

In the simulations, the cases where the Condorcet rule fails to chooses a winner are ignored. In order for Condorcet to choose single winners we run the experiment only for odd values of $n$. On the other hand, Plurality can anytime choose multiple winners. In that case, we require any one of the Plurality winners to coincide with the Condorcet winner.

Here is the procedure followed for the experiment: Given $n$ and $m$, we let GenerateRoot $[\mathrm{m}, \mathrm{n}]$ generate a random root from the uniform probability. If the generated root profile $P$ does not have a Condorcet winner, then we simply ignore it and generate another root. For each root that does have a Condorcet winner, say $a_{i}$, we check and see if it is also chosen by plurality. For this we consider the first row of the preference profile $P$ and make sure that $a_{i}$ occurs in this row at least as many times as every $a_{j}$, for $1 \leq j \leq m$. The ratio of the number of roots in which the Condorcet winner is also a plurality winner to the total number of roots generated which have Condorcet winners is an approximation to the probability that a Condorcet winner is also a plurality winner.

For the case of $n=9$ voters and $m=6$ alternatives, we generated $N=10000$ roots with Condorcet winners from the uniform distribution on the roots. Out of the 10000, the Condorcet 
winners were also plurality winners in 4635 cases. Thus 0.4635 is an approximation to the probability that a Condorcet winner is also a plurality winner for 9 voters and 6 alternatives.

A plot of these probabilities for various $n$ and $m$ computed by using $N=1000$ Condorcet winners for each case appears in Figure 5.

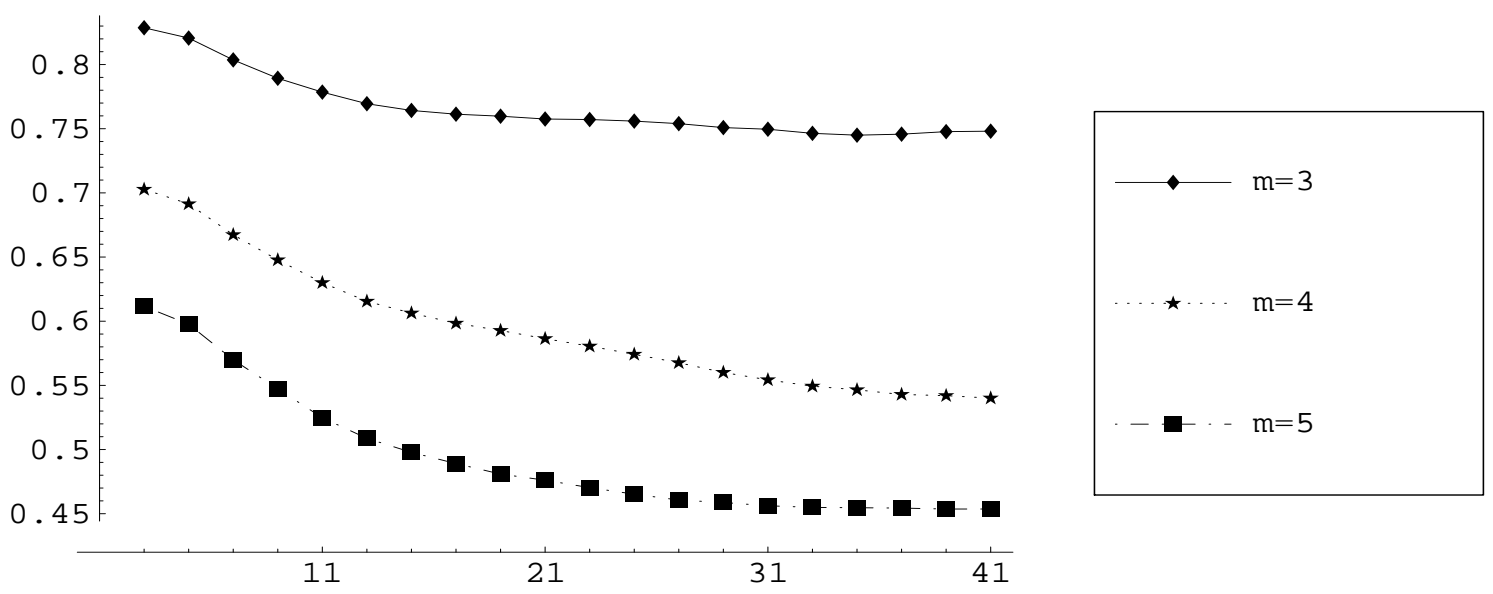

Figure 5: The probability of the Condorcet winners to also be plurality winners in the IANC model after the data has been smoothed out by a 5 -term moving-average filter. The horizontal axis is the number of voters $n$, through odd integers from 3 to 41 . The number of samples used is $N=1000$ per $m / n$ pair.

Figure 6 is the probability of the existence of Condorcet winners for $m=3$ alternatives and various values of number of voters $n$ in the IC, IAC and IANC models. In Table 4 , we give a comparison of the probabilities of the existence of Condorcet winners in the models IC, IAC, and IANC for various $m$. The IC and IAC probabilities are from Gehrlein [8]. The IANC probabilities were computed by generating $=10000$ random roots by GenerateRoot $[\mathrm{m}, \mathrm{n}]$ for each $m / n$ pair. We note that for the very special case of $n=3$ voters, the behavior of the three models shown in Table 4 concerning the probability of the existence of Condorcet winners for increasing number of alternatives is quite similar.

\section{Concluding remarks}

The way that anonymous and neutral SCRs aggregate individual preferences into social choice is independent of the names of the alternatives and of the voters. What matters for these rules is the preference structure in the society of concern, i.e. the relative positions of the alternatives in the 


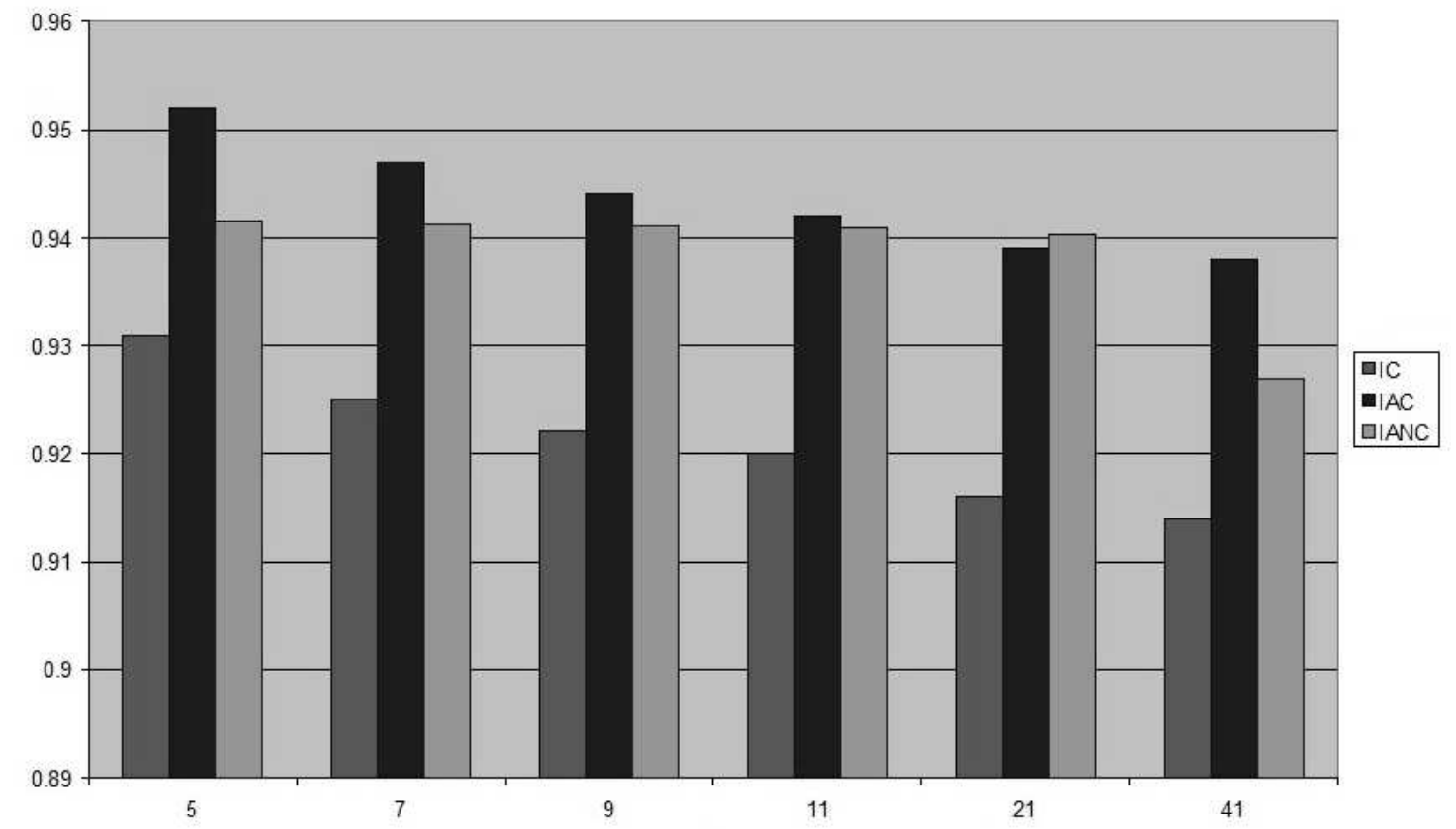

Figure 6: The probability of the existence of Condorcet winners for $m=3$ alternatives and various values of number of voters $n$ in the IC (medium gray), IAC (dark gray), and IANC (light gray) models.

\begin{tabular}{|l|lll|}
\hline & \multicolumn{3}{|c|}{$n=3$} \\
\hline$m$ & IC & IAC & IANC \\
\hline 3 & 0.9444 & 0.9642 & 0.8959 \\
4 & 0.8888 & 0.9015 & 0.8949 \\
5 & 0.8400 & 0.8439 & 0.8384 \\
6 & 0.7977 & 0.7986 & 0.8003 \\
7 & 0.7612 & 0.7613 & 0.7642 \\
8 & 0.7292 & 0.7292 & 0.7259 \\
9 & 0.7010 & 0.7010 & 0.7040 \\
10 & 0.6760 & 0.6760 & 0.6813 \\
11 & 0.6535 & 0.6535 & 0.6529 \\
\hline
\end{tabular}

Table 4: The probability of a Condorcet winner in the IC, IAC, and IANC models for $n=3$ voters. IC and IAC probabilities are from Gehrlein [8]. $N=10000$ samples were generated for the IANC computations. 
preference rankings of individual voters. In this paper, we have used group theory to formulate the structure of the preference structures (roots) for given $m$ and $n$, and presented the ingredients of a symbolic algebra package for the generation of the roots from the uniform distribution to introduce IANC, a probability model to sample voters' preferences.

IANC allows for a sophisticated testbed that can be used to provide a simulative analysis of the behaviors of the anonymous and neutral SCRs with respect to varying number of alternatives and voters. Since, for $m$ alternatives and $n$ voters, all preference profile can be generated from a root by permuting the names of the alternatives and/or voters, the results implemented by an anonymous and neutral SCR remain the same for any of these profiles. Hence, it is important to observe the behaviour of such SCRs as the "core" of the possible preference structures are sampled. Even though probabilistic experiments with the IANC model can be carried out for relatively large values of the parameters, number of voters/alternatives in the thousands or millions would still present computational problems. However, we believe that the combinatorial interpretation of the roots and the resulting formula for $R(m, n)$ may be exploited to derive asymptotic results in certain cases. It is likely that the asymptotic results would be same or similar to the asymptotics of IA and IAC.

Although we introduce the formulation of roots and the IANC model in the framework of social choice, various applications of the approach can be adopted in in other fields. For instance, in a sociometric context, we can assume that each of $n$ individuals ranks $m$ group members (i.e. including ego) on some dimension. Then, an ANEC refers to the family of potential "anonymized" role structures for this system, where one is concerned not with who is specifically being evaluated (or evaluating), but the underlying patterns of evaluation per se. Among the prospects for further research, we believe that theories can be build to predict the role theoretic properties of the system, even where one cannot effectively predict the ratings among specific individuals.

\section{Acknowledgments.}

The authors would like to thank the anonymous referee whose insightful comments and suggestions greatly improved the presentation of this paper.

\section{References}

[1] Berg, S. and D. Lepelley, "On Probability Models in Voting Theory." Statistica Neerlandica 48:133-146, 1994.

[2] Dixon, J. and H. Wilf, "The random selection of unlabeled graphs." Journal of Algorithms, 4:205-212, 1983. 
[3] Eğecioğlu, Ö., "Monte-Carlo Algorithms for Uniform Generation of Anonymous and Neutral Preference Structures." Technical Report TR2005-25, Department of Computer Science, UCSB, July 2005, also in Monte Carlo Methods and Applications, 15:241-255, 2009.

[4] Eğecioğlu, Ö., GenerateRoot[m,n], Mathematica function: available online as a Mathematica notebook http://www.cs.ucsb.edu/ omer/randomvote.nb, Department of Computer Science, UCSB, June 2004.

[5] Feller, W., An Introduction to Probability Theory and its Applications, 3rd Edition, Wiley, New York, 1957.

[6] Fishburn, P. C. and W. Gehrlein, "Condorcet Paradox and Anonymous Preference Profiles." Public Choice 26:1-18, 1978.

[7] Gehrlein, W. V., "Condorcet's Paradox, the Condorcet Efficiency of the Voting Rules." Mathematica Japonica 45:173-199, 1997.

[8] Gehrlein, W. V. "The probability of a Condorcet winner with a small number of voters." Economics Letters, 59:317-321, 1998.

[9] Giritligil, A. and O. Doğan, "An Impossibility Result on Anonymous and Neutral Social Choice Functions." mimeo., 2004.

[10] Giritligil, A. E. and M. Sertel, "Does Majoritarian Approval Matter in Selecting A Social Choice Rule: An Exploratory Panel Study." Social Choice and Welfare 25:43-73, 2005.

[11] Guilbaud, G. T., "Les théories de l'intéret genéral et le problémelogique de l'agrégation." Economie Appliquée 5:501-584, 1952.

[12] Kerber, A., Algebraic Combinatorics via Finite Group Actions, BI \& Brockhaus, Mannheim, 1991.

[13] Lepelley, D. and F. Valognes, "Voting rules, manipulability and social homogeneity." Public Choice 116:165-184, 2003.

[14] Macdonald, I. G., Symmetric Functions and Hall Polynomials, Oxford University Press, 1998.

[15] Wielandt, H., Finite Permutation Groups, Academic Press, New York, 1964.

[16] Sertel, M. and A. E. Giritligil Kara, "Selecting A Social Choice Rule: An Exploratory Panel Study." Sertel, M.R. and Koray, S. (eds.), Advances in Economic Design Springer-Verlag, Heidelberg, 2003.

[17] Condorcet, M. de, Essai sur l'application de l'analyse la probabilit des dcisions rendues la pluralit des voix, Paris, 1785. 